\title{
Trait Somatic Anxiety is Associated With Reduced Directed Exploration and Underestimation of Uncertainty
}

\author{
Haoxue Fan ${ }^{1}$, Samuel J. Gershman ${ }^{1,2}$, and Elizabeth A. Phelps ${ }^{1,2}$ \\ ${ }^{1}$ Department of Psychology, Harvard University \\ ${ }^{2}$ Center for Brain Science, Harvard University
}

\begin{abstract}
Author Note
Haoxue Fan (1D https://orcid.org/0000-0003-3967-6457

Samuel J. Gershman (D) https://orcid.org/0000-0002-6546-3298

Elizabeth A. Phelps (1D https://orcid.org/0000-0002-6215-8159

De-identified behavioral and self-report data as well as analysis code have been made publicly available via the Open Science Framework and can be assessed at https://osf.io/y6urc/?view only=a9cf3daa9c1e4e69bdc79f6d00175fb8. The preregistration form for Experiment1 is available at https://aspredicted.org/BB2 1HT. Early versions of this work were presented at the 2021 Wisconsin Symposium on Emotion, 2021 virtual Society of Neuroeconomics conference, and seminars at Harvard University. We thank Dr. Thomas Rusch for his help with data analysis. We thank members of Phelps Lab and Gershman Lab for
\end{abstract}


insightful discussions. This work was supported by the National Institute on Drug Abuse (Grant No. R01 DA042955) received by E.A.Phelps. The authors declare no conflicts of interest.

Author Contributions: H.Fan, S.J.Gershman, and E.A.Phelps developed the study concept and designed the study. H.Fan collected data and performed data analysis. H.Fan interpreted the data under the supervision of S.J.Gershman and E.A.Phelps. All authors wrote the manuscript and approved its final version for submission.

Correspondence concerning this article should be addressed to Elizabeth A. Phelps, Department of Psychology and Center for Brain Science, Harvard University, 52 Oxford Street, Room 295.10, Cambridge, MA, 02138 E-mail: phelps@fas.harvard.edu 


\begin{abstract}
Exploration is at the core of many real-life decisions, helping people gain information about the environment and make better choices in the long run. Although anxiety has been related to decreased physical exploration and avoidance behavior, past findings on the interaction between anxiety and exploration during decision-making under uncertainty were inconclusive. The current study provides a holistic picture of the anxiety-exploration relationship by focusing on latent factors of trait anxiety and different exploration strategies when facing volatility-induced uncertainty. Across two well-powered online studies $(N=984)$, we demonstrated that people used a hybrid of directed, random, and undirected exploration strategies, which were respectively sensitive to relative uncertainty, total uncertainty, and value difference. The somatic factor of trait anxiety, the propensity to experience physical symptoms of anxiety, was inversely correlated with directed exploration and undirected exploration, manifesting as being less likely to choose the uncertain option and reducing choice stochasticity regardless of uncertainty. Trait somatic anxiety was also related to underestimation of relative uncertainty, which could potentially account for its negative impact on directed exploration. Together, these results reveal the selective role of trait somatic anxiety in modulating both uncertainty-driven and value-driven exploration strategies. More crucially, the differential effects of trait anxiety components underscore the importance of adopting a dimensional approach in future studies. Keywords: Explore-exploit dilemma, Uncertainty, Anxiety, Somatic Anxiety, Reinforcement learning
\end{abstract}




\section{Trait Somatic Anxiety is Associated With Reduced Directed Exploration and Underestimation of Uncertainty}

From daily errands to life decisions, people constantly face the explore-exploit dilemma: should I stick with the current best option (exploit), or should I try something else that could potentially be better (explore)? Because exploration entails inherent risk, it may evoke a heightened level of anxiety, and may be avoided altogether by individuals with chronically high levels of anxiety. However, the multifaceted nature of both exploration and anxiety complicates the study of their relationship. In this paper, we use computational models of exploration in tandem with dimensionality reduction of anxiety measures to cleave the exploration-anxiety relationship into multiple distinct components.

An anticorrelation between anxiety and exploration has long been assumed in animal models (Lister, 1987; Prut \& Belzung, 2003; Zweifel et al., 2011). In the open-field test, a paradigm used to measure exploratory behavior, researchers put the animal in a square box and compare the time it spends in the center and the outer edge of the box. A decrease in time spent in the exposed center area is regarded as a core feature of an anxiety phenotype in non-human animals. Likewise, anxiolytic drugs restore exploratory behavior (Britton \& Britton, 1981). With paradigms mimicking the open-field test and its variants, a few human studies have also shown that anxiety inversely correlates with exploration (Biedermann et al., 2017; Walz et al., 2016).

Beyond the exploration of physical locations, exploration is a key component of human decision making broadly. Specifically, it is a crucial element of tasks examining decision making under uncertainty (Schulz \& Gershman, 2019) in which, to maximize the long-term reward, people need to collect information about a range of options, sometimes at the expense of foregoing the current most rewarding one. Though studies have shown that anxious individuals 
are more averse to uncertainty (Grupe \& Nitschke, 2013), how anxiety influences exploration during decision-making remains unclear. On one hand, people with anxiety symptoms tend to avoid uncertain options (Charpentier et al., 2017), which reduces exploration. On the other hand, anxiety is associated with the elevated valuation of information (Aberg et al., 2021; Bennett et al., 2021), which encourages exploration behavior to reduce uncertainty. It is also possible that the apparently increased exploration reflects an overall tendency to behave more randomly (Aylward et al., 2019).

The inconsistency in these results is due to at least two reasons. First, people engage in distinct forms of exploration given different task structures (e.g., the kind of uncertainty manipulated), making it hard to compare findings across heterogeneous experiments. Second, most existing studies view anxiety as a single-dimension construct, and thus fail to investigate if different anxiety dimensions (e.g., cognitive and somatic anxiety) are linked to distinct exploration patterns. Therefore, the goal of the current study is to experimentally dissociate different exploration strategies in order to investigate their mapping onto distinct anxiety dimensions.

Recent work has revealed that people could use both the value and the uncertainty in the environment to guide exploration. A standard value-driven strategy is softmax exploration (Daw et al., 2006), which chooses an option with probability proportional to the exponential of its expected value. The uncertainty-guided strategies fall into two distinct categories: directed and random exploration (Gershman, 2018; Schwartenbeck et al., 2019; Wilson et al., 2014, 2021). Directed exploration explicitly favors the uncertain option by adding an uncertainty bonus to each option's estimated value. Therefore, choices are directed towards particular options to obtain more information about their value. For instance, when choosing between a new cafe and 
your go-to place for lunch, you may prefer the novel option to assess if it is better than your usual choice. This strategy is sensitive to the relative uncertainty between options (Dubois et al., 2021; Gershman, 2018) and can be captured by reinforcement learning algorithms such as Upper Confidence Bound (Auer et al., 2002; Srinivas et al., 2010). In contrast, random exploration scales choice stochasticity in proportion to the total uncertainty aggregated across options. This kind of exploration can be captured by an algorithm called Thompson sampling (Thompson, 1933). For instance, imagine two cafes that offer different pastries every week (i.e., volatile cafes). Since the pastry menus are constantly updated, the total uncertainty is higher than in a scenario where both cafes offer a fixed set of bakery items (i.e., stable cafes). According to random exploration, people should explore more when facing volatile cafes.

Based on the link between uncertainty and exploration, past work has dissociated directed and random exploration by independently manipulating relative and total uncertainty (Gershman, 2018, 2019; Gershman \& Tzovaras, 2018; Tomov et al., 2020). This was achieved by changing riskiness, that is, the moment-to-moment outcome variability of an option (e.g., the flavor difference of the same pastry between batches). This leaves open the question of whether exploration is truly driven by epistemic uncertainty (as proposed in computational models) or by aleatory uncertainty (the inherent variability of outcomes). To provide data on this question, here we experimentally manipulate epistemic uncertainty while holding aleatory uncertainty fixed. We do this by varying volatility (Piray \& Daw, 2020), that is, the change rate of an option's true value (e.g., the update speed of the pastry menus). Though the uncertainty-driven exploration framework predicts that the impact of uncertainty on exploration is independent of the way it is varied, this hypothesis is yet to be tested. Therefore, the current study examines whether the effects of volatility-induced uncertainty are comparable to those of riskiness-induced uncertainty. 
Focusing on volatility-related uncertainty also helps link the current study with the broader literature on the relationship between anxiety and decision-making, where anxiety is associated with impaired performance as well as biased estimation of uncertainty in environments with changing volatility (Beltzer et al., 2019; Browning et al., 2015; Gagne et al., 2020; Hein et al., 2021; Huang et al., 2017; Pulcu \& Browning, 2017, 2019). To pursue this connection more directly, we report a second study in which we measure uncertainty estimates directly and link them to anxiety dimensions.

Similar to exploration, anxiety is a multifactorial construct. There is considerable variation among individuals in the symptom profiles when experiencing anxiety (Wall \& Lee, 2021), the majority of which can be sorted into cognitive and somatic categories (DeGood \& Tait, 1987; Ree et al., 2008). The cognitive dimension includes symptoms associated with thought processes, including rumination, worry and intrusive thoughts. In contrast, the somatic dimension includes physical manifestations of anxiety such as sweating, trembling, and palpitation. Recent work showed that cognitive and somatic components of anxiety have differential impacts on state transition learning and aversive learning (Sharp et al., 2020; Wise \& Dolan, 2020). Therefore, it is possible that analogous individual differences could mediate exploratory behavior.

\section{Experiment 1}

Experiment 1 dissected exploration strategies people employed during decision-making under volatility-induced uncertainty. Additionally, we assessed the relationship between latent factors of trait anxiety and exploration.

\section{Method}


We report how we determined our sample size, all data exclusions, all manipulations, and all measures for both experiments in the study. All data and analysis code for both experiments are available online (see Data vailability statement). The experiment design, sample size, exclusion criteria, and primary data analysis plan for Experiment 1 were preregistered at https://aspredicted.org/BB2_1HT.

\section{Participants}

We conducted a power analysis by simulating the pilot data, which revealed that a sample size of 500 (after exclusion) would be necessary to detect an effect with $80 \%$ power. Five hundred and thirty-one participants were recruited via the Prolific platform. The informed consent was given before testing. As preregistered, participants were excluded if they reported age outside the range of $18-65$, chose the more rewarding option $<60 \%$ of trials, or did not complete anxiety-related questionnaires. Five hundred and one subjects were included in the final analysis (219 women, 277 men, 5 unreported; Age $M=36.1$ years, $S D=10.9$ ). Participants were paid $\$ 7$ and could earn a bonus up to $\$ 3$ depending on their performance. The study was approved by the Harvard University Committee on the Use of Human Subjects and conformed to American Psychological Association ethical standards.

\section{Experiment Design}

Participants performed 30 blocks of a two-armed bandit task adapted from Gershman (2019). Each block consists of 10 trials. On each trial, participants repeatedly chose between two options ("arms") and received feedback (points delivered by the selected machine) (Figure 1a). They were instructed to collect as many points as possible. Before making a decision, participants saw the labels of the two arms, each of which could be either "Stable" (S) or "Fluctuating" (F). Both types of arms delivered rewards (rounded to the nearest integer) drawn 
from a Gaussian distribution (variance $\tau^{2}=1$ ) around its current generative mean. The generative mean of the fluctuating arm diffused in a Gaussian random walk, that is, its mean on trial $t+$ 1 was drawn from a Gaussian distribution (variance $\tau_{\text {mean }}^{2}(F)=4$ ) centered on its mean on trial $t$. In contrast, the mean of the stable arm was fixed within a block, that is, the variance of the mean $\tau_{\text {mean }}^{2}(S)$ is 0 (see Figure $1 \mathrm{~b}$ for an example). The trial type was denoted by the pair of option labels (e.g., on SF trials, option 1 on the left is stable, and option 2 on the right is fluctuating), resulting in four trial types (SF, FS, SS, and FF). The trial type remained the same within a block and varied randomly across block (i.e., participants encountered a new pair of arms in each block). At the beginning of each block, the generative means of both arms were reset, drawing randomly from a Gaussian distribution (variance $\tau_{0}^{2}=100$ ) with mean 0 . Participants learned the statistics from instructions and had a chance to practice before the formal experiment (see Supplementary Methods for details). The task was programmed using jsPsych library in javascript. A demo can be found online: https://9kapbf3ddlo.cognition.run. 

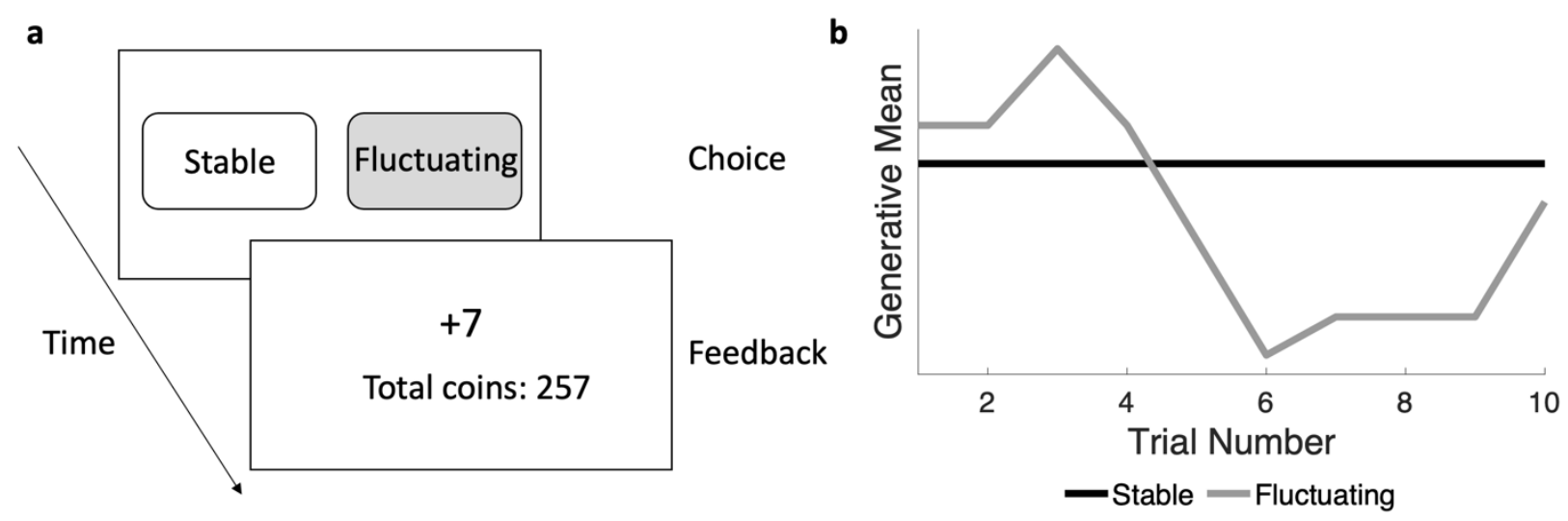

Figure 1 Bandit Task Trial Schematic and Reward Structure (a) On each trial, participants chose between two slot machines and received feedback upon choices. (b)An example reward structure for a stable and a fluctuating arms. In the experiment, the means of both options were resampled from the zero-mean Gaussian at the beginning of each block.

\section{Belief Update Process}

In line with previous literature (Daw et al., 2006; Gershman, 2018, 2019; Speekenbrink \& Konstantinidis, 2015), we assume that participants approximate an ideal Bayesian observer and track both the expected value and uncertainty in the estimation. Given the Gaussian distributional structure underlying our task, the posterior over the value of arm $k$ is Gaussian with mean $Q_{t}(k)$ and variance $\sigma_{t}^{2}(k)$. Both can be recursively updated on each trial $t$ using the Kalman filtering equations:

$$
\begin{gathered}
Q_{t+1}\left(a_{t}\right)=Q_{t}\left(a_{t}\right)+a_{t}\left(r_{t}-Q_{t}\left(a_{t}\right)\right) \\
\sigma_{t+1}^{2}(k)=\sigma_{t}^{2}(k)-\alpha_{t} \sigma_{t}^{2}(k)+\tau_{\text {mean }}^{2}(k) \text { if arm } k \text { is chosen, } \\
=\sigma_{t}^{2}(k)+\tau_{\text {mean }}^{2}(k) \text { if arm } k \text { is not chosen, }
\end{gathered}
$$

where $\alpha_{t}$ is the chosen arm ( $\alpha_{t}=1$ if the left arm is chosen, $\alpha_{t}=2$ if the right arm is chosen), $r_{t}$ is the delivered reward, and the Kalman gain $\alpha_{t}$ is given by: 


$$
\alpha_{t}=\left(\sigma_{t}^{2}(k)+\tau_{\text {mean }}^{2}(k)\right) /\left(\sigma_{t}^{2}(k)+\tau_{\text {mean }}^{2}(k)+\tau^{2}\left(a_{t}\right)\right)
$$

Note that the posterior mean is updated only for the chosen arm regardless of its type, whereas the posterior variance for the fluctuating arm is updated every trial irrespective of the participant's choice. The diffusion noise for the fluctuating arm $\tau_{\text {mean }}{ }^{2}(F)$ and for the stable arm

$\tau_{\text {mean }}^{2}(S)$ are set to 4 and 0 , respectively. The posterior means are initialized with the prior mean, $Q_{1}(k)=0$ for all $k$, and posterior variances are initialized with the prior variance, $\sigma_{1}^{2}(k)=\tau_{0}^{2}=$ 100. Kalman filtering is an idealization of learning from noisy observations, and past research has shown that it can account for human choices in bandit tasks well (Daw et al., 2006; Speekenbrink \& Konstantinidis, 2015).

\section{Choice Probability Analysis}

Participants' data were pooled across conditions and modeled using the following probit regression model:

$$
P\left(a_{t}=1 \mid \mathbf{w}\right)=\Phi\left(w_{1} \mathrm{~V}_{t}+w_{2} \mathrm{RU}_{t}+w_{3} \mathrm{~V}_{t} / \mathrm{TU}_{t}\right),
$$

where $\mathrm{V}_{t}=Q_{t}(1)-Q_{t}(2)$ is the value difference between two options, $\mathrm{RU}_{t}=\sigma_{t}(1)-\sigma_{t}(2)$ denotes the relative uncertainty, $\mathrm{TU}_{t}=\sqrt{ }\left(\sigma_{t}^{2}(1)+\sigma_{t}^{2}(2)\right)$ denotes the total uncertainty, and $\Phi(\bullet)$ is the standard Gaussian cumulative distribution function. The value estimate and estimation uncertainty were obtained using Kalman filtering. Past work (Gershman, 2018) proved that this is the exact analytic form of a hybrid of Thompson Sampling and Upper Confidence Bound algorithms. A positive $w_{2}$ means that people add an uncertainty bonus to an option's value proportional to its relative uncertainty, which directs exploration towards the option they are more uncertain about. In contrast, a positive $w_{3}$ indicates that as the total uncertainty in the environment goes up, people increase choice randomness accordingly. Specifically, if $w_{2}=0$, the model is insensitive to random uncertainty and is reduced to pure random exploration. If $w_{3}=0$, 
the model is insensitive to total uncertainty and is reduced to pure directed exploration. Finally, if $w_{2}=w_{3}=0$, the model is only influenced by the value of options, and the strategy is similar to softmax exploration.

To obtain a descriptive characterization of choice behavior across conditions, we also modeled choices as a function of experimental condition (SF, FS, SS, FF):

$$
P\left(a_{t}=1 \mid \mathbf{w}\right)=\Phi\left(\sum_{j} w_{4}{ }^{j} \pi_{t j}+w_{5}{ }^{j} \pi_{t j} \mathrm{~V}_{t}\right)
$$

where $j$ is the experimental condition, and $\pi_{t j}=1$ if trial $t$ belongs to condition $j,=0$ otherwise. We refer to the $w_{4}$ terms as intercepts and the $w_{5}$ terms as slopes in the following sections. The parameters were estimated using generalized linear mixed-effect models in Matlab (version R2020a). All $t$-tests and $F$-tests for fixed effect coefficients are two-sided.

\section{Trait Anxiety Measure}

We administered two trait anxiety scales to capture its multidimensionality: State-Trait Anxiety Inventory trait scale (STAI-T; Spielberger, 1983) and State-Trait Inventory for Cognitive and Somatic Anxiety trait scale (STICSA-T; Ree et al., 2008), which were filled out after the behavioral task (other scales administered were reported in Supplementary Methods). STAI-T is the most widely used trait anxiety measure (Knowles \& Olatunji, 2020) and has a high internal consistency (Cronbach's $\alpha=.90$; Spielberger, 1983). Compared to STAI-T, STICSA-T includes more somatic items and has superior divergent validity (Roberts et al., 2016). To extract latent factors of trait anxiety and reduce dimensionality, we conducted an exploratory factor analysis (EFA) on all items from STICSA-T and STAI-T $(N=82)$. The number of factors was selected based on parallel analysis, which compares the eigenvalues generated from the data matrix to those generated from a simulated matrix created from random data of the same size and retains the eigenvalues that are above the $95 \%$ quantile of the simulated ones (Horn, 1965). 
Factor scores were then calculated using the Bartlett method (Bartlett, 1937) implemented in the psych package (Revelle, 2021) in R (version 4.0.2; R Core Team, 2020).

\section{Modeling the Effects of Trait Anxiety Factors on Exploration Strategies}

We extended model (1) to include trait anxiety factor scores and their interactions with V, RU and V/TU:

$$
P\left(a_{t}=1 \mid \mathbf{w}\right)=\Phi\left(w_{1} \mathrm{~V}_{t}+w_{2} \mathrm{RU}_{t}+w_{3} \mathrm{~V}_{t} / \mathrm{TU}_{t}+\sum_{n}\left(w_{4}{ }^{n} \mathrm{~V}_{t}: \mathrm{Anx}^{n}+w_{5}{ }^{n} \mathrm{RU}_{t}: \mathrm{Anx}^{n}+w_{6}{ }^{n} \mathrm{~V}_{t} / \mathrm{TU}_{t}: \mathrm{Anx}^{n}+w_{7}{ }^{n} \mathrm{Anx}^{n}\right)\right),
$$

where $n$ denotes the $n^{\text {th }}$ trait anxiety factor. The factor scores were standardized before entering the model. Age and gender were also entered as covariates. A positive $w_{5}$ indicates that people scoring high on the trait anxiety factor are more sensitive to relative uncertainty and engage in more directed exploration. Similarly, a positive $w_{6}$ implies elevated random exploration (i.e., behaving more randomly when facing the same total uncertainty). On the other hand, a positive $w_{4}$ is interpreted as a negative association between the trait anxiety factor and undirected exploration due to an increased sensitivity to the value difference between options. The parameters were estimated using generalized linear mixed-effect models in Matlab.

\section{Data Availability}

All de-identified data are publicly available at the Open Science Framework website: https://osf.io/y6urc/?view_only=a9cf3daa9c1e4e69bdc79f6d00175fb8 (Fan et al., 2021). This repository includes the behavioral data, code used to generate regression models, code used to generate figures, as well as links to the preregistration and the task demo.

\section{Code Availability}

Code used to fit belief update model, generate regression models and generate figures are available at the Open Science Framework

https://osf.io/y6urc/?view_only=a9cf3daa9c1e4e69bdc79f6d00175fb8 (Fan et al., 2021).

\section{Results}




\section{People Use a Hybrid of Directed and Random Exploration Strategies}

The current experimental design allows us to independently manipulate relative and total uncertainty to investigate their separate influences on different exploration strategies. Because the volatility of the fluctuating arm is higher than the stable arm, the fluctuating arm is more uncertain. If directed exploration is, as hypothesized, sensitive to relative uncertainty, people should prefer option 1 (the left arm) in condition FS and option 2 (the right arm) in condition SF, even the value difference suggests otherwise. This is equivalent to an increase in the intercept of condition FS vs. SF (Figure 2a). It is worth noting that the comparison between condition FS and SF holds the total uncertainty constant because there is always one fluctuating arm and one stable arm.

A comparison between conditions SS and FF reveals how people respond to a shift in total uncertainty $(\mathrm{FF}>\mathrm{SS})$ while holding the relative uncertainty constant. If random exploration is sensitive to total uncertainty, it should predict that people behave more randomly in condition FF. This can be reflected as an increase in slope in the psychometric curve for condition FF vs. SS., that is, the probability of choosing option 1 is closer to random $(p=0.5)$ in condition FF (Figure 2b). 

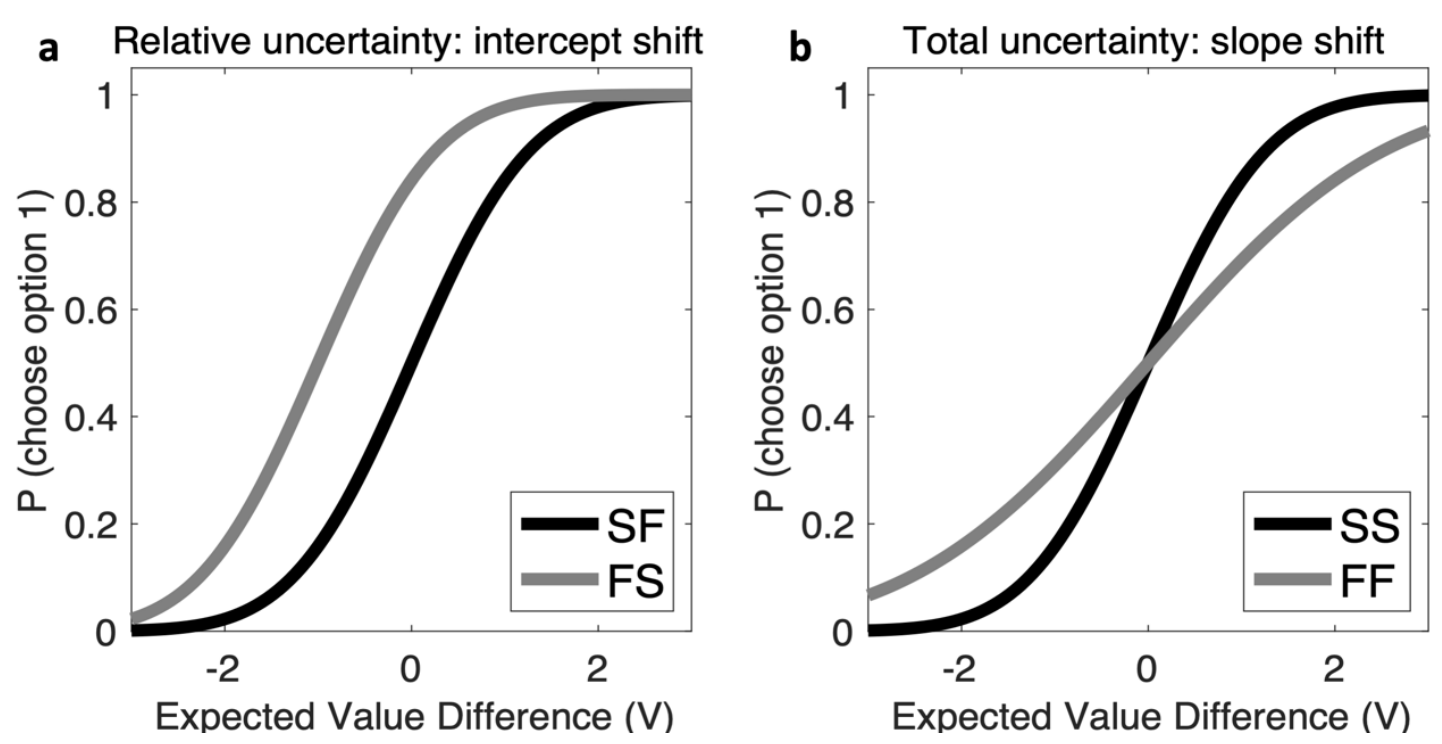

Figure 2 Predictions of Choice Probability Function Change Across Conditions. (a) Directed exploration predicts a preference for the uncertain option, which manifests as a shift in intercept in opposite directions for SF and FS trials. (b) Random exploration predicts more choice stochasticity when total uncertainty is high, equivalent to a steeper curve for FF trials than SS trials.

Our data suggest that people performed well in the task, choosing the most rewarding option on average $79.76 \%$ ( $S E=5.22 \%$ ) of the time (see Supplementary Figure 1a). In line with our predictions, there was a significant intercept shift between condition (FS and SF: $F(1,150292)=6.20, p=.013)$, showing that people direct their exploration towards the uncertain option (Figure 3a, Supplementary Figure 2a). Additionally, the intercept of SF is significantly negative $(B=-0.045,95 \%$ CI $[-0.077,-0.014], t(150292)=-2.82, p=.005$, two-sided $)$, indicating that people formed a bias towards choosing option 2 when its relative uncertainty was high. The intercept of FS was not significantly different from zero $(B=0.016,95 \%$ CI $[-0.015$, 0.047], $t(150292)=1.01, p=.312)$. There was no significant difference between intercept for 
condition FF and SS $(F(1,150292)=0.06, p=.814)$, suggesting that people adjusted directed exploration according to relative uncertainty but not total uncertainty.

We also found evidence for random exploration, manifesting as a steeper curve for FF than SS $(F(1,150292)=81.07, p<.001$; Figure 3b, Supplementary Figure 3b). This suggests that people chose more randomly when facing high total uncertainty. There was no significant difference between slope SF vs. FS $(F(1,150292)=2.77, p=.096)$, corroborating that random exploration is sensitive to total uncertainty, not relative uncertainty.
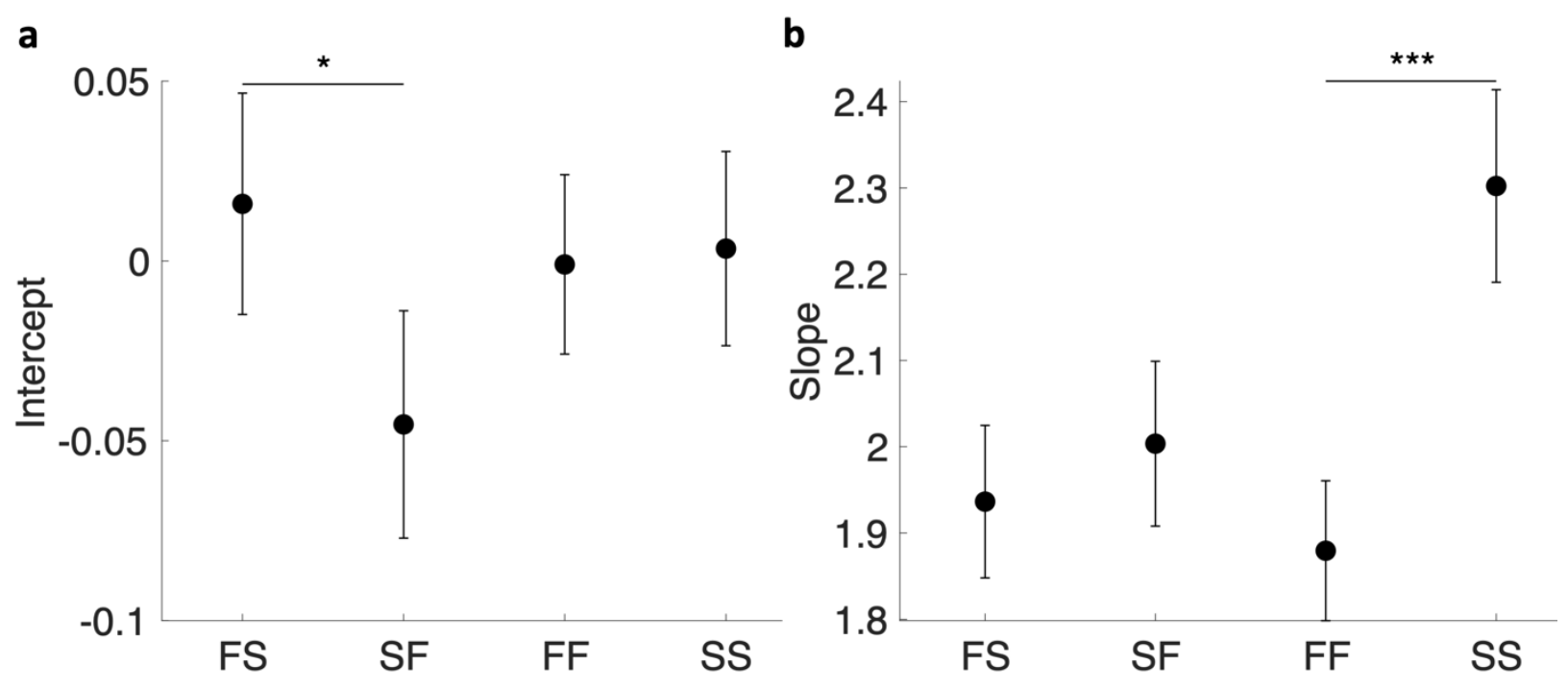

Figure 3 Probit Regression Results (Experiment 1) (a) The intercept of FS trials was larger than that of SF trials, while the intercepts of FF and SS trials did not differ. (b) The slope of FF trials was larger than that of SS trials, while the slopes of SF and FS trials did not differ. Error bars are $95 \%$ confidence intervals. $*: p<.05 . * *: p<.001$. 
On a trial-by-trial basis, we found that people were sensitive to RU, V/TU, and V, with their fixed effect coefficients all significantly larger than zero (RU: $B=0.137,95 \%$ CI $[0.092$, $0.183], t(150297)=5.89 ; \mathrm{V} / \mathrm{TU}: B=1.060,95 \% \mathrm{CI}[0.930,1.190], t(150297)=15.98 ; \mathrm{V}: B=$ $1.576,95 \%$ CI $[1.486,1.666], t(150297)=34.43$; all $p \mathrm{~s}<.001)$. The model with three regressors also outperformed model candidates that only allowed for one exploration strategy (see Supplementary Table 2). Together, our data spoke to the hypothesis that people used a hybrid of directed and random exploration strategies, which are respectively sensitive to relative and total uncertainty induced by volatility.

\section{Trait Somatic Anxiety is Associated With Reduced Directed Exploration and Choice}

\section{Stochasticity}

The EFA resulted in a four-factor structure, which was validated with the confirmatory factor analysis in an independent sample $(N=797$, see Supplementary Methods and Results for details). Based on the item loadings (Figure 4, Supplementary Table 3), we labeled the four factors as "Somatic Anxiety" (subjective experience of physiological symptoms), "Cognitive Anxiety" (worrying thoughts and rumination), "Negative Affect" (lack of positive affective experience), and "Low Self-esteem" (negative self-image). 

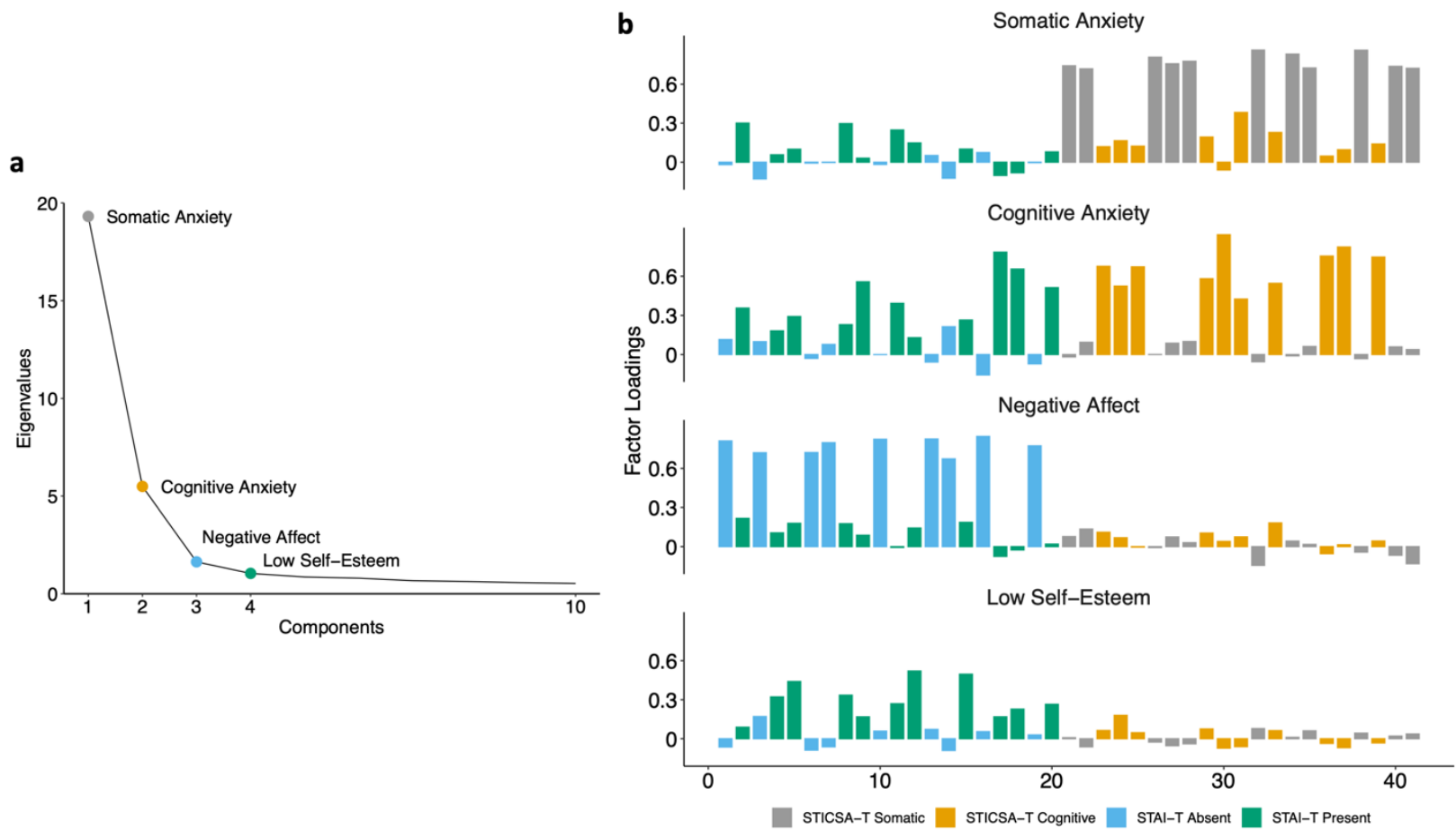

Figure 4 Exploratory Factor Analysis Results. (a) Scree plot of eigenvalues. (b) Factor loadings of items on trait anxiety factors. Items from different subscales are distinguished by their colors. STICSA-T Somatic $=$ somatic subscale of STICSA-T; STICSA-T Cognitive $=$ cognitive subscale of STICSA-T; STAI-T Absent = STAI-T anxiety absent items; STAI-T Present $=$ STAI-T anxiety present items.

The Somatic Anxiety factor had two distinct effects on exploration strategies. First, its interaction with random uncertainty was significantly negative $(B=-0.070,95 \%$ CI $[-0.134,-$ 0.006], $t(150273)=-2.12, p=.034$; Figure 5). In other words, given the same level of relative uncertainty, people high on trait somatic anxiety were less likely to engage in directed exploration. Second, the Somatic Anxiety factor was associated with increased sensitivity to value difference between options $(B=0.217,95 \%$ CI $[0.089,0.345], t(150273)=3.32, p$ $<.001$ ), indicating reduced undirected exploration. These effects were robust to different ways of 
trait somatic anxiety measurement, remaining significant when trait somatic anxiety was quantified by raw STICSA-T somatic subscale score (SI Table 6). We also found a positive relationship between the Negative Affect factor and directed exploration $(B=0.077,95 \% \mathrm{CI}$ $[0.026,0.129], t(150273)=2.93, p=.003$; Figure 5). However, this effect was not replicated in Experiment 2 (see Experiment 2 Results Section). No trait anxiety factor significantly influenced random exploration, and neither the Cognitive Anxiety nor the Low Self-esteem factor interacted with any exploration strategies (all $p \mathrm{~s}>.05$; Table 1 ). 


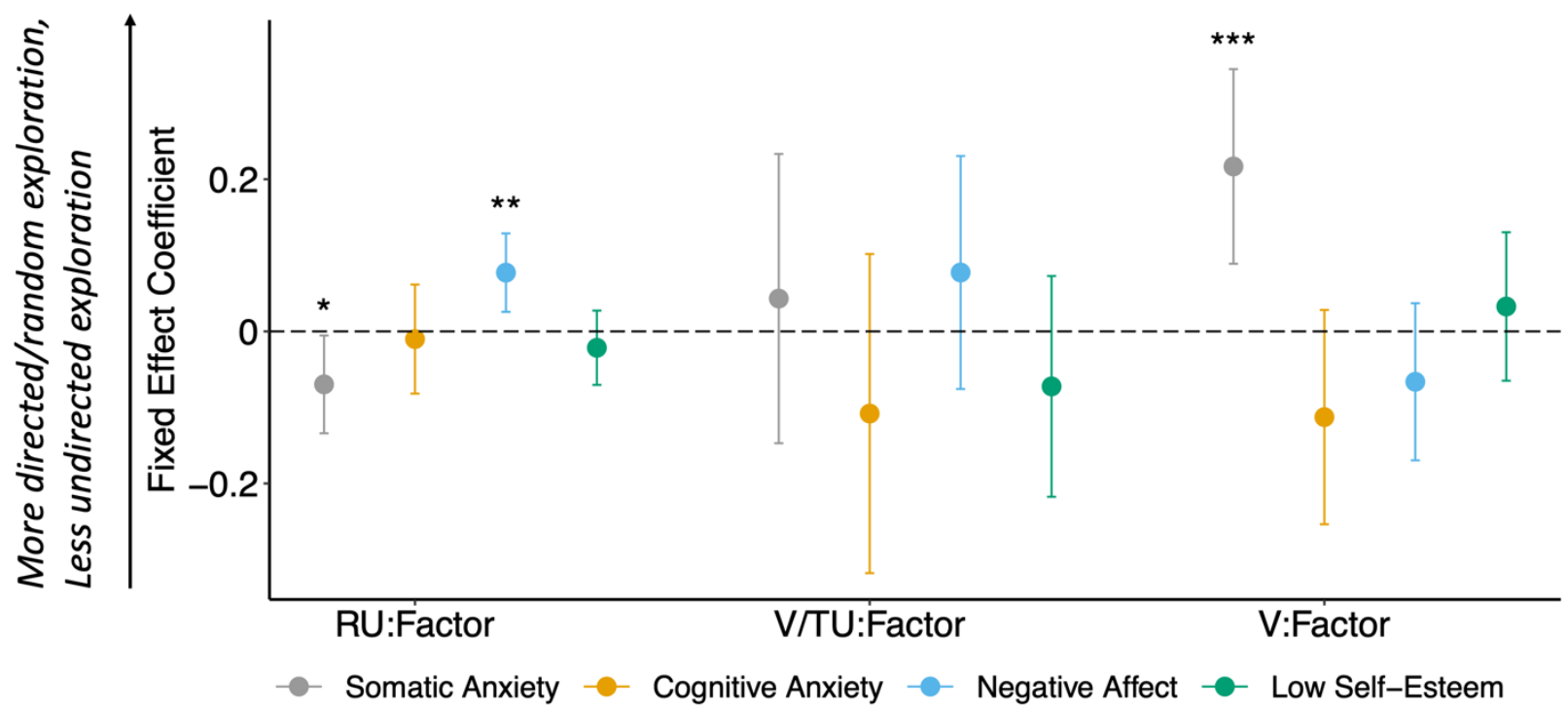

Figure 5 Effects of Trait Anxiety Factors on Exploration Strategies (Experiment 1). Factor scores were obtained using EFA results in Experiment 1. All factors were standardized and entered into the same model together with age and gender. Points indicate the fixed effect coefficients for each predictor, while error bars represent the $95 \%$ confidence interval. A positive coefficient for RU:Factor (V/TU:Factor) indicates increased directed (random) exploration. A positive coefficient for V:Factor indicates decreased undirected exploration. ${ }^{*}: p<.05 .{ }^{* *}: p$ $<.01 .{ }^{* * *}: p<.001$. 


\section{Table 1}

Interactions between Trait Anxiety Factors on Exploration Strategies

\begin{tabular}{lccccccccc}
\hline \multicolumn{1}{c}{$\begin{array}{c}\text { Trait anxiety } \\
\text { factor }\end{array}$} & \multicolumn{3}{c}{ RU:Factor } & \multicolumn{9}{c}{ V/TU:Factor } & \multicolumn{3}{c}{ V:Factor } \\
\cline { 2 - 10 } & \multicolumn{1}{c}{$B(S E)$} & $t$ & $p$ & $B(S E)$ & $t$ & $p$ & $B(S E)$ & $t$ & $p$ \\
\hline $\begin{array}{l}\text { Experiment 1 }(\mathrm{N}=501) \\
\text { Somatic Anxiety }\end{array}$ & $-0.070(0.033)$ & -2.12 & $.034^{*}$ & $0.043(0.097)$ & 0.45 & .657 & $0.217(0.065)$ & 3.32 & $<.001^{* * *}$ \\
Cognitive & $-0.010(0.037)$ & -0.28 & .782 & $-0.108(0.107)$ & -1.01 & .313 & $-0.113(0.072)$ & -1.57 & .117 \\
Anxiety & & & & & & & & & \\
Negative Affect & $0.077(0.026)$ & 2.93 & $.003^{* *}$ & $0.077(0.078)$ & 0.99 & .322 & $-0.066(0.053)$ & -1.26 & .208 \\
Low Self-esteem & $-0.022(0.025)$ & -0.86 & .387 & $-0.072(0.074)$ & -0.98 & .328 & $0.033(0.05)$ & 0.66 & .512 \\
\hline Experiment 2 (N $=484)$ & & & & & & & & \\
Somatic Anxiety & $-0.050(0.023)$ & -2.14 & $.032^{*}$ & $0.098(0.086)$ & 1.14 & .254 & $0.194(0.048)$ & 4.05 & $<.001^{* * *}$ \\
Cognitive & $-0.005(0.027)$ & -0.20 & .842 & $-0.093(0.096)$ & -0.97 & .332 & $-0.052(0.054)$ & -0.97 & .331 \\
Anxiety & & & & & & & & \\
Negative Affect & $0.010(0.022)$ & 0.44 & .662 & $-0.035(0.079)$ & -0.44 & .661 & $-0.102(0.044)$ & -2.32 & $.020^{*}$ \\
Low Self-esteem & $-0.011(0.021)$ & -0.52 & .602 & $0.075(0.073)$ & 1.04 & .300 & $0.032(0.041)$ & 0.79 & .428 \\
\hline
\end{tabular}

Note. Factor scores were obtained using EFA results in Experiment 1. All trait anxiety factors were standardized and entered into the same model together with age and gender. A positive coefficient for RU:Factor (V/TU:Factor) indicates increased directed (random) exploration. A positive coefficient for $\mathrm{V}$ :Factor indicates decreased undirected exploration. ${ }^{*}: p<.05 .{ }^{* *}: p$ $<.01{ }^{* * *}: p<.001$

\section{Experiment 2}

In Experiment 1, we documented an inverse relationship between trait somatic anxiety and directed and undirected exploration. It remained unclear whether the reduced exploration was attributed to a change in the process of learning (underestimated RU and/or overestimated V) or decision-making (accurate value and uncertainty estimates but reduced sensitivity towards 
RU and/or increased sensitivity towards V), or both. In Experiment 2, we investigated this question using a reward prediction task. We tested directly whether trait somatic anxiety is related to a systematic change in the subjective calculation of $\mathrm{RU}$ and/or $\mathrm{V}$, which are crucial to directed and undirected exploration, respectively.

\section{Method}

\section{Participants}

We intended to get a sample size comparable to that in Experiment 1 and recruited 556 participants via the Prolific platform. The informed consent was given prior to testing. The exclusion criteria were the same as Experiment 1. Four hundred and eighty-four subjects were included in the final analysis of the bandit task (197 women, 279 men, 8 unreported; Age $M=$ 35.3 years, $S D=10.5$ ). We additionally excluded prediction trials in which the reward prediction $>50$ or $<-50$ (the maximum and minimum possible experienced reward in the task was 47 and -44). This resulted in excluding $10.52 \%$ of all trials. Subjects who always selected the same trial-by-trial confidence rating were also excluded ( 29 subjects, $4.03 \%$ of all trials). Four hundred and forty-six subjects were included in the final analysis of the prediction task (190 women, 248 men, 8 unreported; Age $M=34.6$ years, $S D=10.4$ ). Participants were paid $\$ 15$ and could earn a bonus up to $\$ 4$, with $\$ 3$ depending on their performance in bandit task and $\$ 1$ depending on their reward prediction accuracy. The study was approved by the Harvard University Committee on the Use of Human Subjects and conformed to American Psychological Association ethical standards.

\section{Experiment Design}

Participants performed the two-armed bandit task for 30 blocks with 10 trials in each block. The two-armed bandit task was similar to that used in Experiment 1, except that the 
participants completed a prediction task at the end of each block (Figure 6). During the prediction task, participants guessed how many points one machine will generate in the next trial by entering an integer (see Supplementary Methods for details). Feedback on their prediction was not provided. They then rated their confidence in their reward prediction on a scale from 0 (guess randomly) to 10 (very confident). Participants were encouraged to use the full range of the scale. After the prediction task, participants encountered two new slot machines and started a new block of the bandit task. Whether they made a prediction for the left or the right arm first is randomized. At the end of the experiment, one prediction task trial was randomly chosen. If the prediction made on that trial was within one of the real generative mean value of the arm, the participant received a $\$ 1$ bonus, which is in addition to the bonus depending on their performance (maximum \$3).

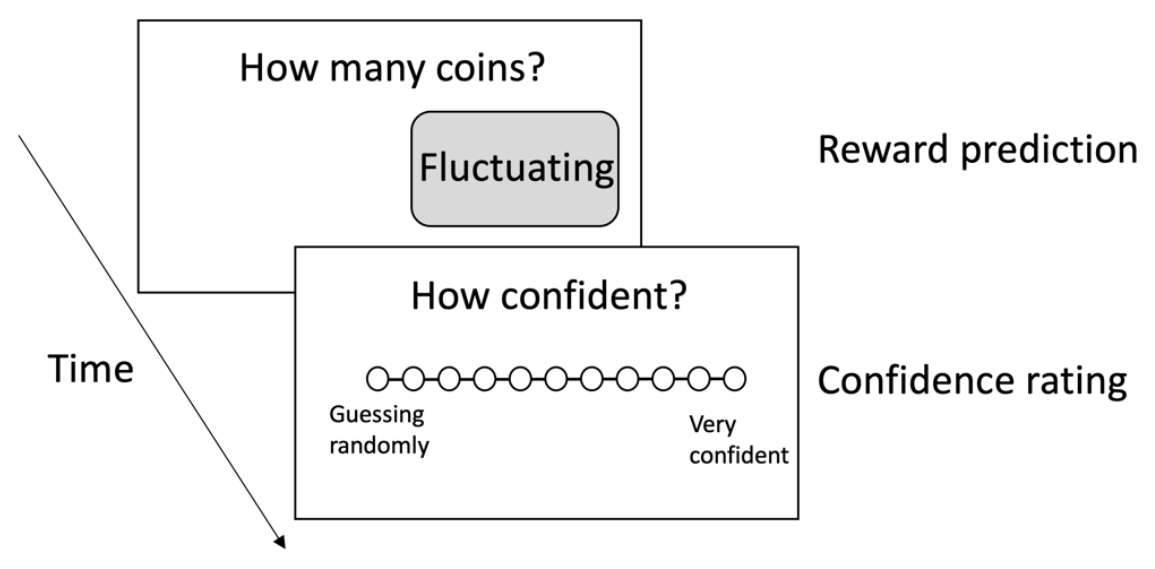

Figure 6 Prediction Task Trial Schematic. The prediction task was completed at the end of each block. 


\section{Trait Anxiety Measure}

Consistent with Experiment 1, STAI-T and STICSA-T were administered as trait anxiety measures. Latent factors of trait anxiety were extracted using the factor loading structure obtained in Experiment 1.

\section{Subjective Value and Uncertainty Estimates}

We treated participants' reward prediction as their subjective value estimation and their confidence rating as the inverse of their uncertainty estimation (i.e., we assume a high confidence level implies low estimation uncertainty). Because directed and undirected exploration strategies are sensitive to RU and V, we focused on the subjective estimates of RU and V (see Supplementary Methods and Results for a discussion on TU). The subjective counterpart of RU and V were defined as:

Subjective V = option 1's reward prediction - option 2's reward prediction,

Subjective RU = option 2's confidence - option 1's confidence.

\section{Modeling the Effects of Trait Anxiety Factors on Subjective Value and Uncertainty Estimates}

We used the following linear mixed-effect regressions to examine how trait anxiety factors modulate the relationship between subjective and normative estimates of $\mathrm{V}$ and RU:

$$
\begin{gathered}
\text { Subjective } \mathrm{V}_{t}=w_{1} \mathrm{~V}_{t}+\sum_{n}\left(w_{2}{ }^{n} \mathrm{~V}_{t}: \mathrm{Anx}^{n}+w_{3} \mathrm{Anx}^{n}\right), \\
\text { Subjective } \mathrm{RU}_{t}=w_{1} \mathrm{RU}_{t}+\sum_{n}\left(w_{2}{ }^{n} \mathrm{RU}_{t}: \mathrm{Anx}^{n}+w_{3} \mathrm{Anx}^{n}\right)
\end{gathered}
$$

The factor scores were standardized and age and gender were entered as covariates. Across two equations, a positive $w_{2}$ means that given the same normative estimates of $\mathrm{V}(\mathrm{RU})$, participants high on the trait anxiety factor report higher subjective $\mathrm{V}(\mathrm{RU})$, which is equivalent to an overestimation. The parameters were estimated using linear mixed-effect models in Matlab.

\section{Results}

\section{Replicating Main Results of Experiment 1}


First, we repeated the analysis in Experiment 1 to make sure that adding the prediction task did not interfere with the exploration strategies people adopted in the two-armed bandit task. Indeed, the manipulation of relative and total uncertainty causally influenced uncertainty-driven exploration: people directed their exploration towards the relatively uncertain option $(F(1$, $144892)=10.17, p=.001 ;$ Figure 7a, Supplementary Figure 2c). In addition, people chose more randomly when the total uncertainty was high $(F(1,144892)=31.23, p<.001$; Figure $7 \mathrm{~b}$, Supplementary Figure 2d). Examining participants" behavior on a trial-by-trial basis, the model including V, RU, and VTU fit participants' behavior better than models that only allowed for one exploration strategy (see Supplementary Table 2), again showing that people used a hybrid of undirected, directed and random exploration $(\mathrm{V}: B=1.153,95 \% \mathrm{CI}[1.081,1.225], t(144897)=$ 31.39; RU: $B=0.209,95 \%$ CI $[0.175,0.247], t(144897)=11.36$; V/TU: $B=1.053,95 \% \mathrm{CI}$ $[0.938,1.185], t(144897)=16.85 ;$ all $p \mathrm{~s}<.001)$. 

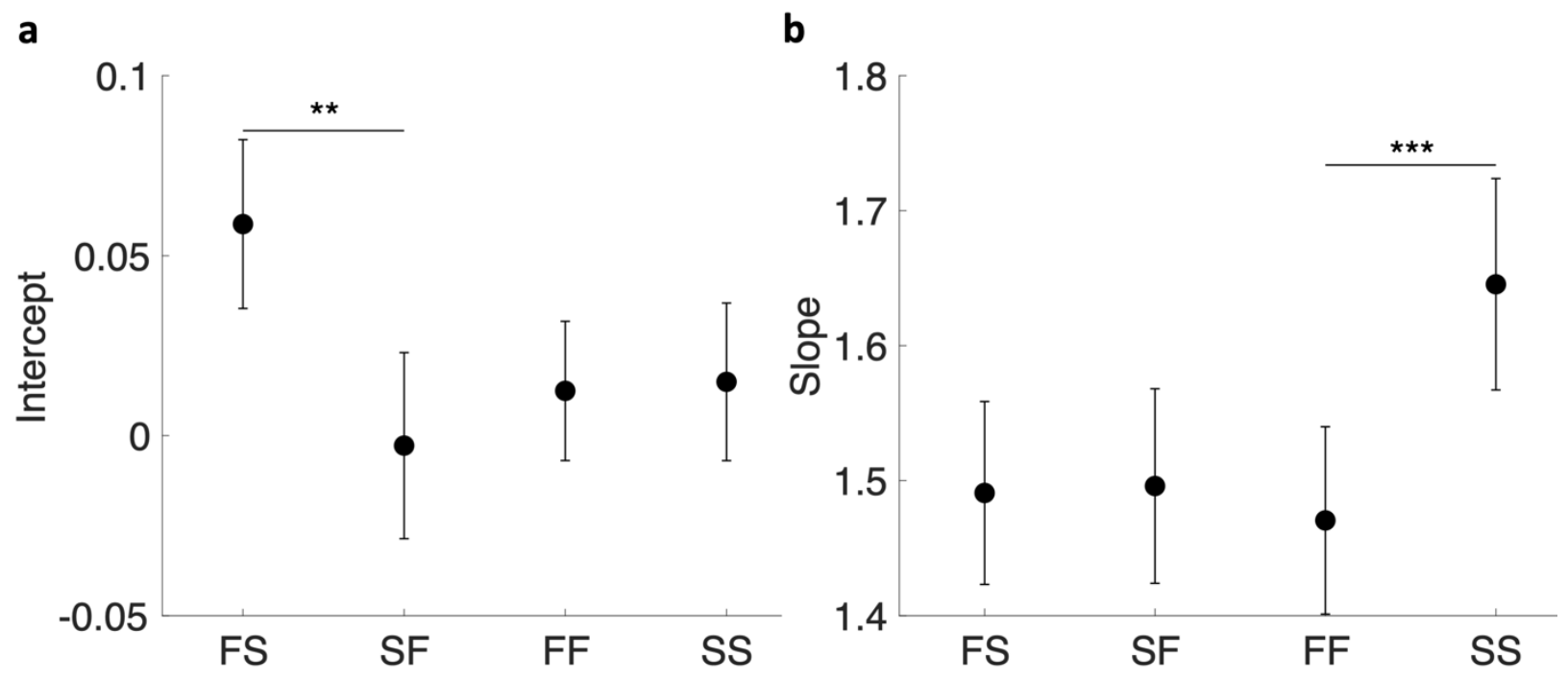

Figure 7 Probit Regression Results (Experiment 2). (a)The intercept of FS trials was larger than that of SF trials, while the intercepts of FF and SS trials did not differ. (b)The slope of FF trials was larger than that of SS trials, while the slopes of SF and FS trials did not differ. Error bars are $95 \%$ confidence intervals. ${ }^{* *}: p<.01 .{ }^{* * *}: p<.001$.

Second, we replicated the inverse relationship between trait somatic anxiety and exploration. On the one hand, people high on the Somatic Anxiety factor were less likely to direct exploration towards the option with high relative uncertainty $(B=-0.050,95 \%$ CI $[-0.096$, -0.004], $t(145173)=-2.14, p=.032$; Figure 8, Table 1). On the other hand, they demonstrated a lower level of choice stochasticity, choosing the option with a higher expected value more often $(B=0.194,95 \%$ CI $[0.100,0.289], t(145173)=4.05, p<.001)$. The effects of Negative Affect factor on exploration are inconsistent between Experiment 1 and Experiment 2: Experiment 2 data did not support a relationship between Negative Affect and directed exploration $(B=0.010$, $95 \%$ CI $[-0.033,0.053], t(145173)=0.44, p=.662)$. Instead, there was a positive association 
between Negative Affect and undirected exploration $(B=-0.102,95 \%$ CI $[-0.188,-0.016]$, $t(145173)=-2.32, p=.020)$.

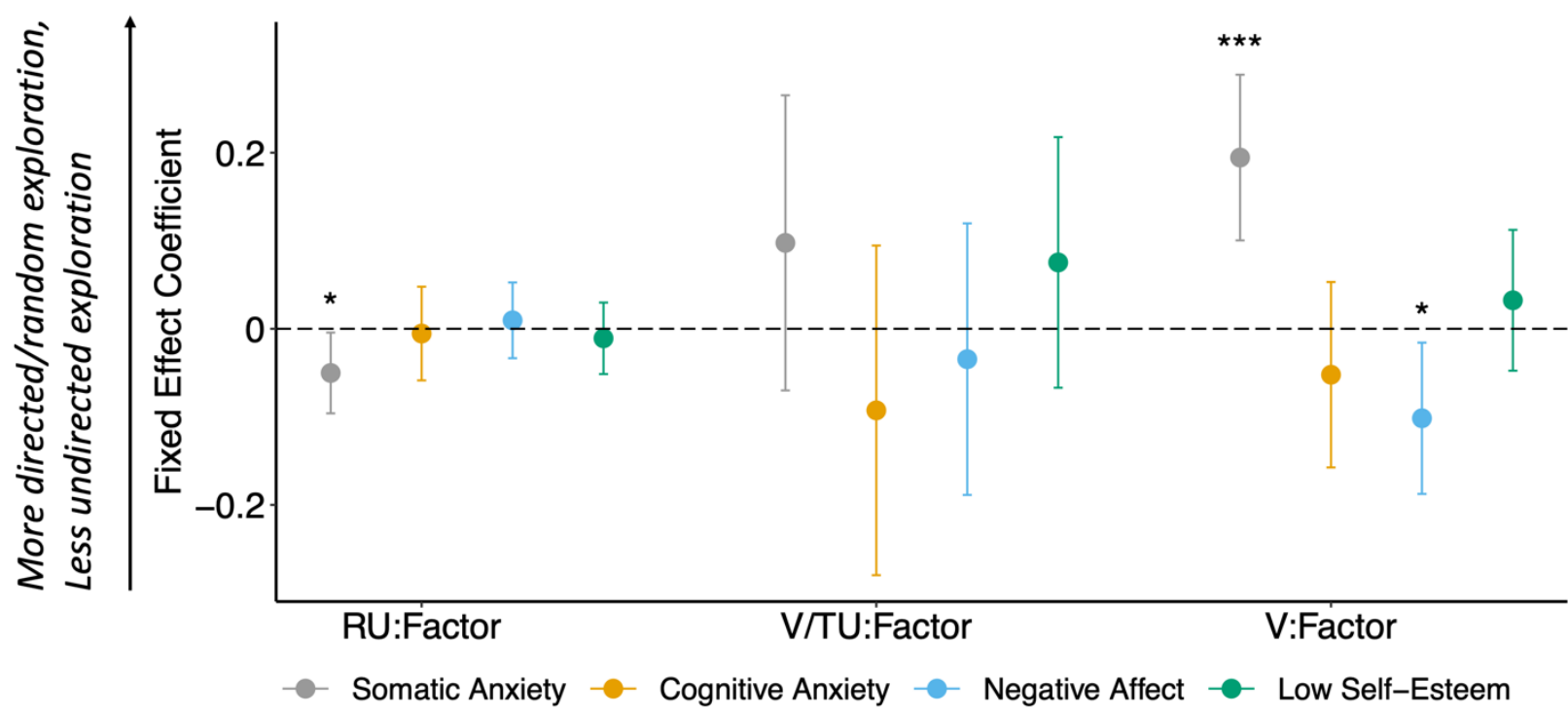

Figure 8 Effects of Trait Anxiety Factors on Exploration Strategies (Experiment 2). Factor scores were obtained using EFA results in Experiment 1. All trait anxiety factors were standardized and entered into the same model together with age and gender. Points indicate the fixed effect coefficients for each predictor, while error bars represent the $95 \%$ confidence interval. A positive coefficient for RU:Factor (V/TU:Factor) indicates increased directed (random) exploration. A positive coefficient for V:Factor indicates decreased undirected exploration. $*: p<.05 . * * *: p<.001$. 


\section{Subjective Value and Uncertainty Track the Normative Estimates}

In the reward prediction task, self-report reward predictions and confidence ratings tracked the normative value and uncertainty estimates well (Reward prediction vs. Posterior mean: $r_{\mathrm{s}}=.72, p<.001$; Confidence vs. Posterior $\left.S D: r_{\mathrm{s}}=-.24, p<.001\right)$. The inverse relationship between normative uncertainty estimate and confidence is in line with past work showing that given Bayesian confidence hypothesis (Aitchison et al., 2015; Fleming \& Daw, 2017), confidence for the Gaussian estimation problem is monotonically related to the inverse posterior $S D$ (Xiang et al., 2021). The subjective $\mathrm{V}$ and RU also significantly correlated with their normative counterparts (V: $\left.r_{\mathrm{s}}=.78, p<.001 ; \mathrm{RU}: r_{\mathrm{s}}=.42, p<.001\right)$.

\section{Trait Somatic Anxiety is Associated With Underestimation of Relative Uncertainty}

Somatic Anxiety factor of trait anxiety interacted negatively with normative estimate of relative uncertainty $(B=-0.293,95 \% \mathrm{CI}[-0.411,-0.174], t(12076)=-4.85, p<.001$; see Table 2), suggesting that people high on trait somatic anxiety tend to underestimate relative uncertainty in the environment. The effect of underestimation remained significant when using STICSA-T somatic subscale as trait somatic anxiety measure $(B=-0.211,95 \%$ CI $[-0.309,-0.112], t(12082)$ $=-4.20, p<.001$; see Supplementary Table 5). This could be a potential explanation of the diminished directed exploration in high trait somatic anxious individuals. There was also a significantly negative interaction between Somatic Anxiety and normative estimate of value difference $(B=-1.435,95 \%$ CI $[-2.035,-0.246], t(12076)=-2.50, p=.012)$, implying a tendency to underestimate value difference among those scoring high on Somatic Anxiety factor. We failed to detect this interaction when measuring trait somatic anxiety with STICSA-T somatic subscale $(B=-0.532,95 \% \mathrm{CI}[-1.270,0.206], t(12082)=-1.41, p=.158)$. 


\section{Table 2}

The Relationship Between Trait Anxiety Factors and Subjective RU and V Estimates

\begin{tabular}{lcccccc}
\hline \multicolumn{1}{c}{$\begin{array}{c}\text { Trait anxiety } \\
\text { factor }\end{array}$} & \multicolumn{3}{c}{ RU:Factor } \\
& \multicolumn{1}{c}{$B(\mathrm{SE})$} & $\mathrm{t}$ & $p$ & $B(\mathrm{SE})$ & $t$ & $p$ \\
\cline { 2 - 7 } & $-0.293(0.06)$ & -4.85 & $<.001^{* * *}$ & $-1.141(0.456)$ & -2.50 & $.012^{*}$ \\
\hline Somatic Anxiety & $0.087(0.076)$ & 1.15 & .249 & $0.697(0.564)$ & 1.24 & .217 \\
Cognitive Anxiety & 0.15 :Factor & \\
Negative Affect & $0.186(0.065)$ & 2.86 & $.004^{* *}$ & $0.575(0.488)$ & 1.18 & .239 \\
Low Self-esteem & $-0.084(0.06)$ & -1.41 & .159 & $0.06(0.444)$ & 0.134 & .893 \\
\hline
\end{tabular}

Note. Factor scores were obtained using EFA results in Experiment 1. All trait anxiety factors were standardized and entered into the same model together with age and gender. The definition of subjective RU and V can be found in Experiment 2 Result Section. A positive RU:Factor (V:Factor) indicates an overestimation of RU (V). ${ }^{*}: p<.05 . * *: p<.01 . * * *: p<.001$.

\section{Discussion}

The current study examined the association between trait anxiety components and exploration during decision-making under uncertainty. Across two large-scale online experiments, we found a selective role of trait somatic anxiety, the propensity to experience enduring physical symptoms of anxiety, in modulating exploration in two distinct ways: first, trait somatic anxious individuals showed diminished directed exploration, being less likely to direct exploration towards the more uncertain option. The reward prediction task data offered a potential mechanistic account for this inverse relationship, showing that people high on trait somatic anxiety tended to underestimate relative uncertainty. Second, people high on trait somatic anxiety exhibited a low level of undirected exploration, choosing less randomly 
regardless of uncertainty. Interestingly, none of the other trait anxiety components interact with any exploration strategy.

To dissociate and quantify exploration strategies, we manipulated different kinds of uncertainty in a two-armed bandit task. Dovetailing with previous work (Gershman, 2019; Gershman \& Tzovaras, 2018; Tomov et al., 2020), we found behavioral signatures for directed, random and undirected exploration, which were driven by relative uncertainty, total uncertainty, and value difference, respectively. Specifically, the current study altered uncertainty by changing volatility (i.e., the diffuse rate of an option's generative mean), while holding the riskiness of both options fixed (i.e., the variance of the outcome distribution). This design removed the potential interference effect of risk aversion. In other words, people may be reluctant to try an uncertain option due to a desire to avoid risk, therefore offering a cleaner measure of directed and random exploration. More importantly, these findings extend the uncertainty-driven exploration framework by proving that people are sensitive to epistemic uncertainty in their posterior estimates of the choices instead of aleatoric uncertainty caused merely by outcome variability.

The negative association between trait somatic anxiety and directed exploration is consistent with previous work showing that anxious individuals find uncertainty more aversive and tend to avoid it (Charpentier et al., 2017; Grupe \& Nitschke, 2013). In a volatile environment, this avoidance behavior is self-reinforcing. As time goes by, the unchosen option grows more uncertain because the information obtained from the last observation is less predictive of its current value, which further drives a somatic anxious individual away from it. In the long term, insufficient directed exploration impedes people from collecting information and updating the value of the uncertain options, which could lead to suboptimal decision strategies 
and give rise to real-life maladaptive avoidance behavior. Therefore, reduced directed exploration can be seen as a cognitive risk factor for the later development of anxiety disorders. On the other hand, diminished undirected exploration suggests that people scoring high on trait somatic anxiety made choices in line with the value difference. In tandem with other cognitive biases, declined indirect exploration could also lead to avoidance behavior. For example, suppose an anxious individual holds a pessimistic belief towards novel objects and assigns low initial value to them (Zorowitz et al., 2020), they may tend to avoid the unfamiliar options without a single interaction.

Complementary to avoiding the uncertain option, trait somatic anxiety was linked to a reduced ability to track relative uncertainty with self-report confidence. In other words, when holding the uncertainty level of one option unchanged, an increase in the uncertainty level of the other option transforms to a smaller change in confidence difference between two options in somatic anxious individuals. This altered representation of uncertainty converges with previous research showing that state anxious individuals hold a more precise belief about the reward contingencies in the environment and update less (Hein et al., 2021). Moreover, recent work uniquely linked the somatic component of anxiety to smaller uncertainty estimate during aversive learning while the direction of the relationship was reversed for cognitive anxiety (Wise \& Dolan, 2020). Building upon these findings, the current study further showed that somatic anxious individuals were poor at sensing the uncertainty difference between options. The blunted response to uncertainty could serve as an explanation for the reduced directed exploration: an anxious person may favor the uncertain option less due to a smaller uncertainty bonus, which is proportional to subjective relative uncertainty. However, the current data could not directly test this hypothesis because confidence ratings were only acquired at the end of each block instead of 
on a trial-by-trial basis. The task was designed this way due to the concern that eliciting reward prediction and confidence rating too often would interfere with the exploration process. Future studies could use different paradigms to examine whether subjective relative uncertainty mediates the relationship between trait somatic anxiety and directed exploration.

The current study utilizes a dimensional approach to examine the interaction between anxiety and exploration. Among all latent factors of trait anxiety identified in the present study, only Somatic Anxiety was associated with a change in exploration. In contrast, the others did not reliably modulate the exploration process. These differential effects are critical in understanding the inconsistencies in past work on the anxiety-exploration relationship. Most existing studies treated trait anxiety as a unitary construct while their measurements might tap onto distinct components. According to our factor structure, STAI-T primarily reflects the Negative Affect factor, which was associated with an increase in directed exploration in Experiment 1. This finding matches the recent work by Aberg et al. (2021). However, this relationship disappeared in Experiment 2 when the reward prediction task was added, implying that the effect of Negative Affect might not be robust to task modification. Another recent study (Zaller et al., 2021) administered Penn State Worry Questionnaire (Meyer et al., 1990), which primarily measures cognitive symptoms of anxiety (Sharp et al., 2015) and failed to detect a relationship between anxiety and directed exploration. This finding aligns well with our finding that trait cognitive anxiety did not interact with exploration. On the other hand, past literature on physical exploratory behavior focused on patients diagnosed with panic disorder or agoraphobia (Walz et al., 2016), both of which are anxiety disorders primarily characterized by elevated somatic symptoms (American Psychiatric Association, 2013). Specifically, Kallai et al. (2007) found that compared to those diagnosed with generalized anxiety disorder, people who have panic disorder 
with agoraphobia demonstrated more disturbed exploratory behavior, hinting at a unique contribution of somatic anxiety to suppressed spatial exploration. Taken together, our dimensional approach showed the promise of unifying past work on exploration across task domains.

An important question remains unanswered: why does trait somatic anxiety interact with exploration? Despite the prevalence of somatic symptoms in anxiety disorders, it is only recently that researchers began to examine its specific impact on learning and decision-making in the general population (Gillan et al., 2020; Sharp et al., 2020; Wise \& Dolan, 2020). Drawing inspirations from work on panic disorder, we conjecture that altered exploration in trait somatic anxiety could be attributed to enhanced interoception, that is, increased sensitivity to arousal signals (Domschke et al., 2010; Ehlers, 1991; Yoris et al., 2015). These signals are important because arousal systems are involved in the processing of uncertainty and value, which could be used to guide decisions (de Berker et al., 2016; FeldmanHall et al., 2019; Miu et al., 2008; Nassar et al., 2012). Heightened interoception in somatic anxious individuals might amplify the impact of physiological arousal on exploration (Lenow et al., 2017), which could account for the altered subjective computation of relative uncertainty in the same population. It is left for future studies to provide a holistic picture of the dynamics between somatic anxiety, physiological and subjective arousal, and exploration.

This study has a few limitations. Although we proved the specificity of the associations between trait somatic anxiety and exploration strategies by controlling for other trait anxiety components in the same analysis, we do not know whether these effects are robust to including other psychiatric symptoms such as compulsivity. We believe that our results will still hold since no clear relationship between compulsive behavior and exploration was documented in past 
literature. That said, given the high comorbidity of obsessive-compulsive disorder and anxiety (Nestadt et al., 2009), it is important for future studies to take compulsivity into account so as to obtain cleaner measures of the effects of anxiety on decision-making components. Besides, the current study examined the anxiety-exploration relationship in an online general population where the distribution of trait somatic anxiety was positively skewed. Therefore, future work should test the generalizability of our findings in a clinically diagnosed population such as panic disorder patients who usually demonstrate more somatic symptoms. 


\section{References}

Aberg, K. C., Toren, I., \& Paz, R. (2021). A neural and behavioral tradeoff underlies exploratory decisions in normative anxiety. BioRxiv. https://doi.org/10.1101/2020.09.16.300525

Aitchison, L., Bang, D., Bahrami, B., \& Latham, P. E. (2015). Doubly Bayesian analysis of confidence in perceptual decision-making. PLoS Computational Biology, 11(10), e1004519. https://doi.org/10.1371/journal.pcbi.1004519

American Psychiatric Association. (2013). Diagnostic and statistical manual of mental disorders (5th ed.). https://doi.org/10.1176/appi.books.9780890425596

Auer, P., Cesa-Bianchi, N., \& Fischer, P. (2002). Finite-time analysis of the multiarmed bandit problem. Machine Learning, 47, 235-256. https://doi.org/10.1023/A:1013689704352

Aylward, J., Valton, V., Ahn, W.-Y., Bond, R. L., Dayan, P., Roiser, J. P., \& Robinson, O. J. (2019). Altered learning under uncertainty in unmedicated mood and anxiety disorders. Nature Human Behaviour, 3(10), 1116-1123. https://doi.org/10.1038/s41562-019-0628-0

Bartlett, M. S. (1937). The statistical conception of mental factors. British Journal of Psychology, 28(1), 97. https://doi.org/10.1111/j.2044-8295.1937.tb00863.x

Beltzer, M. L., Adams, S., Beling, P. A., \& Teachman, B. A. (2019). Social Anxiety and Dynamic Social Reinforcement Learning in a Volatile Environment. Clinical Psychological Science, 7(6), 1372-1388. https://doi.org/10.1177/2167702619858425

Bennett, D., Sutcliffe, K., Tan, N. P.-J., Smillie, L. D., \& Bode, S. (2021). Anxious and obsessive-compulsive traits are independently associated with valuation of noninstrumental information. Journal of Experimental Psychology: General, 150(4), 739-755. https://doi.org/10.1037/xge0000966 
Biedermann, S. V., Biedermann, D. G., Wenzlaff, F., Kurjak, T., Nouri, S., Auer, M. K., Wiedemann, K., Briken, P., Haaker, J., Lonsdorf, T. B., \& Fuss, J. (2017). An elevated plus-maze in mixed reality for studying human anxiety-related behavior. BMC Biology, 15(1), 125. https://doi.org/10.1186/s12915-017-0463-6

Britton, D. R., \& Britton, K. T. (1981). A sensitive open field measure of anxiolytic drug activity. Pharmacology Biochemistry and Behavior, 15(4), 577-582. https://doi.org/10.1016/0091-3057(81)90212-4

Browning, M., Behrens, T. E., Jocham, G., O’Reilly, J. X., \& Bishop, S. J. (2015). Anxious individuals have difficulty learning the causal statistics of aversive environments. Nature Neuroscience, 18(4), 590-596. https://doi.org/10.1038/nn.3961

Charpentier, C. J., Aylward, J., Roiser, J. P., \& Robinson, O. J. (2017). Enhanced Risk Aversion, But Not Loss Aversion, in Unmedicated Pathological Anxiety. Biological Psychiatry, 81(12), 1014-1022. https://doi.org/10.1016/j.biopsych.2016.12.010

Daw, N. D., O’Doherty, J. P., Dayan, P., Seymour, B., \& Dolan, R. J. (2006). Cortical substrates for exploratory decisions in humans. Nature, 441(7095), 876-879. https://doi.org/10.1038/nature04766

de Berker, A. O., Rutledge, R. B., Mathys, C., Marshall, L., Cross, G. F., Dolan, R. J., \& Bestmann, S. (2016). Computations of uncertainty mediate acute stress responses in humans. Nature Communications, 7(1), 10996. https://doi.org/10.1038/ncomms10996

DeGood, D. E., \& Tait, R. C. (1987). The cognitive-somatic anxiety questionnaire: Psychometric and validity data. Journal of Psychopathology and Behavioral Assessment, 9(1), 75-87. https://doi.org/10.1007/BF00961633 
Domschke, K., Stevens, S., Pfleiderer, B., \& Gerlach, A. L. (2010). Interoceptive sensitivity in anxiety and anxiety disorders: An overview and integration of neurobiological findings. Clinical Psychology Review, 30(1), 1-11. https://doi.org/10.1016/j.cpr.2009.08.008

Dubois, M., Habicht, J., Michely, J., Moran, R., Dolan, R. J., \& Hauser, T. U. (2021). Human complex exploration strategies are enriched by noradrenaline-modulated heuristics. ELife, 10, e59907. https://doi.org/10.7554/eLife.59907

Ehlers, A. (1991). Cognitive factors in panic attacks: Symptom probability and sensitivity. Journal of Cognitive Psychotherapy, 5(3), 157-173. https://psycnet.apa.org/record/199223724-001

Fan, H., Gershman, S. J., \& Phelps, E. A. (2021, August 13). Trait somatic anxiety is associated with reduced directed exploration and underestimation of uncertainty. Retrieved from osf.io/y6urc

FeldmanHall, O., Glimcher, P., Baker, A. L., NYU PROSPEC Collaboration, \& Phelps, E. A. (2019). The Functional Roles of the Amygdala and Prefrontal Cortex in Processing Uncertainty. Journal of Cognitive Neuroscience, 31(11), 1742-1754. https://doi.org/10.1162/jocn_a_01443

Fleming, S. M., \& Daw, N. D. (2017). Self-evaluation of decision-making: A general Bayesian framework for metacognitive computation. Psychological Review, 124(1), 91-114. https://doi.org/10.1037/rev0000045

Gagne, C., Zika, O., Dayan, P., \& Bishop, S. J. (2020). Impaired adaptation of learning to contingency volatility in internalizing psychopathology. Elife, 9, e61387. https://doi.org/10.7554/elife.61387 
Gershman, S. J. (2018). Deconstructing the human algorithms for exploration. Cognition, 173, 34-42. https://doi.org/10.1016/j.cognition.2017.12.014

Gershman, S. J. (2019). Uncertainty and exploration. Decision, 6(3), 277-286. https://doi.org/10.1037/dec0000101

Gershman, S. J., \& Tzovaras, B. G. (2018). Dopaminergic genes are associated with both directed and random exploration. Neuropsychologia, 120, 97-104. https://doi.org/10.1016/j.neuropsychologia.2018.10.009

Gillan, C. M., Kosinski, M., Whelan, R., Phelps, E. A., \& Daw, N. D. (2016). Characterizing a psychiatric symptom dimension related to deficits in goal-directed control. ELife, 5, e11305. https://doi.org/10.7554/eLife.11305

Grupe, D. W., \& Nitschke, J. B. (2013). Uncertainty and anticipation in anxiety: An integrated neurobiological and psychological perspective. Nature Reviews Neuroscience, 14(7), 488-501. https://doi.org/10.1038/nrn3524

Hein, T. P., de Fockert, J., \& Ruiz, M. H. (2021). State anxiety biases estimates of uncertainty and impairs reward learning in volatile environments. NeuroImage, 224, 117424. https://doi.org/10.1016/j.neuroimage.2020.117424

Horn, J. L. (1965). A rationale and test for the number of factors in factor analysis. Psychometrika, 30(2), 179-185. https://doi.org/10.1007/BF02289447

Huang, H., Thompson, W., \& Paulus, M. P. (2017). Computational Dysfunctions in Anxiety: Failure to Differentiate Signal From Noise. Biological Psychiatry, 82(6), 440-446. https://doi.org/10.1016/j.biopsych.2017.07.007 
Knowles, K. A., \& Olatunji, B. O. (2020). Specificity of trait anxiety in anxiety and depression: Meta-analysis of the State-Trait Anxiety Inventory. Clinical Psychology Review, 82, 101928. https://doi.org/10.1016/j.cpr.2020.101928

Lenow, J. K., Constantino, S. M., Daw, N. D., \& Phelps, E. A. (2017). Chronic and acute stress promote overexploitation in serial decision making. Journal of Neuroscience, 37(23), 5681-5689. https://dx.doi.org/10.1523\%2FJNEUROSCI.3618-16.2017

Lister, R. G. (1987). The use of a plus-maze to measure anxiety in the mouse. Psychopharmacology, 92(2), 180-185. https://doi.org/10.1007/bf00177912

Meyer, T. J., Miller, M. L., Metzger, R. L., \& Borkovec, T. D. (1990). Development and validation of the penn state worry questionnaire. Behaviour Research and Therapy, 28(6), 487-495. https://doi.org/10.1016/0005-7967(90)90135-6

Miu, A. C., Heilman, R. M., \& Houser, D. (2008). Anxiety impairs decision-making: Psychophysiological evidence from an Iowa Gambling Task. Biological Psychology, 77(3), 353-358. https://doi.org/10.1016/j.biopsycho.2007.11.010

Nassar, M. R., Rumsey, K. M., Wilson, R. C., Parikh, K., Heasly, B., \& Gold, J. I. (2012). Rational regulation of learning dynamics by pupil-linked arousal systems. Nature Neuroscience, 15(7), 1040-1046. https://doi.org/10.1038/nn.3130

Nestadt, G., Di, C. Z., Riddle, M. A., Grados, M. A., Greenberg, B. D., Fyer, A. J., McCracken, J. T., Rauch, S. L., Murphy, D. L., \& Rasmussen, S. A. (2009). Obsessive-compulsive disorder: Subclassification based on co-morbidity. Psychological Medicine, 39(9), 14911501. https://doi.org/10.1017/s0033291708004753

Piray, P., \& Daw, N. D. (2020). Unpredictability vs. volatility and the control of learning. BioRxiv. https://doi.org/10.1101/2020.10.05.327007 
Prut, L., \& Belzung, C. (2003). The open field as a paradigm to measure the effects of drugs on anxiety-like behaviors: A review. European Journal of Pharmacology, 463(1-3), 3-33. https://doi.org/10.1016/s0014-2999(03)01272-x

Pulcu, E., \& Browning, M. (2017). Affective bias as a rational response to the statistics of rewards and punishments. ELife, 6, e27879. https://doi.org/10.7554/eLife.27879

Pulcu, E., \& Browning, M. (2019). The Misestimation of Uncertainty in Affective Disorders. Trends in Cognitive Sciences, 23(10), 865-875. https://doi.org/10.1016/j.tics.2019.07.007

R Core Team (2020). R: A language and environment for statistical computing. R Foundation for Statistical Computing, Vienna, Austria. URL https://www.R-project.org/.

Ree, M. J., French, D., MacLeod, C., \& Locke, V. (2008). Distinguishing Cognitive and Somatic Dimensions of State and Trait Anxiety: Development and Validation of the State-Trait Inventory for Cognitive and Somatic Anxiety (STICSA). Behavioural and Cognitive Psychotherapy, 36(03). https://doi.org/10.1017/S1352465808004232

Revelle W (2021). psych: Procedures for Psychological, Psychometric, and Personality Research. Northwestern University, Evanston, Illinois. R package version 2.1.6, https://CRAN.R-project.org/package=psych.

Roberts, K. E., Hart, T. A., \& Eastwood, J. D. (2016). Factor structure and validity of the StateTrait Inventory for Cognitive and Somatic Anxiety. Psychological Assessment, 28(2), 134-146. https://doi.org/10.1037/pas0000155

Schulz, E., \& Gershman, S. J. (2019). The algorithmic architecture of exploration in the human brain. Current Opinion in Neurobiology, 55, 7-14. https://doi.org/10.1016/j.conb.2018.11.003 
Schwartenbeck, P., Passecker, J., Hauser, T. U., FitzGerald, T. H., Kronbichler, M., \& Friston, K. J. (2019). Computational mechanisms of curiosity and goal-directed exploration. Elife, 8, e41703. https://doi.org/10.7554/eLife.41703

Sharp, P. B., Dolan, R. J., \& Eldar, E. (2020). Cognitive map learning is disrupted in compulsivity and anxious arousal. https://doi.org/10.31234/osf.io/x29jq

Sharp, P. B., Miller, G. A., \& Heller, W. (2015). Transdiagnostic dimensions of anxiety: Neural mechanisms, executive functions, and new directions. International Journal of Psychophysiology, 98(2), 365-377. https://doi.org/10.1016/j.ijpsycho.2015.07.001

Speekenbrink, M., \& Konstantinidis, E. (2015). Uncertainty and exploration in a restless bandit problem. Topics in Cognitive Science, 7(2), 351-367. https://doi.org/10.1111/tops.12145

Spielberger, C. D. (1983). State-Trait Anxiety Inventory for Adults (STAI-AD) [Database record]. APA PsycTests. https://psycnet.apa.org/doi/10.1037/t06496-000

Srinivas, N., Krause, A., Seeger, M., \& Kakade, S. M. (2010). Gaussian process optimization in the bandit setting: No regret and experimental design. In Proceedings of the 27th international confer- ence on machine learning (pp. 1015-1022). https://arxiv.org/abs/0912.3995

Thompson, W. R. (1933). On the likelihood that one unknown probability exceeds another in view of the evidence of two samples. Biometrika, 25(3/4), 285-294. https://doi.org/10.1093/biomet/25.3-4.285

Tomov, M. S., Truong, V. Q., Hundia, R. A., \& Gershman, S. J. (2020). Dissociable neural correlates of uncertainty underlie different exploration strategies. Nature Communications, 11(1), 2371. https://doi.org/10.1038/s41467-020-15766-z 
Wall, A., \& Lee, E. (2021). What do Anxiety Scales Really Measure? An Item Content Analysis of Self-Report Measures of Anxiety. https://doi.org/10.31234/osf.io/t7gpx

Walz, N., Mühlberger, A., \& Pauli, P. (2016). A human open field test reveals thigmotaxis related to agoraphobic fear. Biological Psychiatry, 80(5), 390-397. https://doi.org/10.1016/j.biopsych.2015.12.016

Wilson, R. C., Bonawitz, E., Costa, V. D., \& Ebitz, R. B. (2021). Balancing exploration and exploitation with information and randomization. Current Opinion in Behavioral Sciences, 38, 49-56. https://doi.org/10.1016/j.cobeha.2020.10.001

Wilson, R. C., Geana, A., White, J. M., Ludvig, E. A., \& Cohen, J. D. (2014). Humans use directed and random exploration to solve the explore-exploit dilemma. Journal of Experimental Psychology: General, 143(6), 2074-2081. https://doi.org/10.1037/a0038199

Wise, T., \& Dolan, R. J. (2020). Associations between aversive learning processes and transdiagnostic psychiatric symptoms in a general population sample. Nature Communications, 11(1), 4179. https://doi.org/10.1038/s41467-020-17977-w

Xiang, Y., Graeber, T., Enke, B., \& Gershman, S. J. (2021). Confidence and central tendency in perceptual judgment. Attention, Perception, \& Psychophysics. https://doi.org/10.3758/s13414-021-02300-6

Yoris, A., Esteves, S., Couto, B., Melloni, M., Kichic, R., Cetkovich, M., Favaloro, R., Moser, J., Manes, F., Ibanez, A., \& Sedeño, L. (2015). The roles of interoceptive sensitivity and metacognitive interoception in panic. Behavioral and Brain Functions, 11(1), 14. https://doi.org/10.1186/s12993-015-0058-8 
Zaller, I., Zorowitz, S., \& Niv, Y. (2021). Information Seeking on the Horizons Task Does Not Predict Anxious Symptomatology. Biological Psychiatry, 89(9), S217-S218.

Zorowitz, S., Momennejad, I., \& Daw, N. D. (2020). Anxiety, avoidance, and sequential evaluation. Computational Psychiatry, 4, 1-17.

https://doi.org/10.1016/j.biopsych.2021.02.550

Zweifel, L. S., Fadok, J. P., Argilli, E., Garelick, M. G., Jones, G. L., Dickerson, T. M. K., Allen, J. M., Mizumori, S. J. Y., Bonci, A., \& Palmiter, R. D. (2011). Activation of dopamine neurons is critical for aversive conditioning and prevention of generalized anxiety. Nature Neuroscience, 14(5), 620-626. https://doi.org/10.1038/nn.2808 